\title{
The transition from localized to homogeneous plasticity during nanoindentation of an amorphous metal
}

\author{
C. A. Schuh $\dagger$, A. S. Argon \\ Massachusetts Institute of Technology, Cambridge, Massachusetts 02139, USA \\ T. G. NIEH \\ Lawrence Livermore National Laboratory, Livermore, California 94550, USA \\ and J. WADSWORTH \\ Battelle Memorial Institute, Columbus, Ohio 43201, USA
}

[Received 30 September 2002 and accepted in revised form 25 March 2003]

\begin{abstract}
Using nanoindentation, we examine the fundamental nature of plasticity in a bulk amorphous metal. We find that the mechanics of plasticity depend strongly on the indentation loading rate, with low rates promoting discretization of plasticity into rapid bursts. For sufficiently slow indentations, we find that plastic deformation becomes completely discretized in a series of isolated yielding events. As the loading rate is increased, a transition from discrete to continuous yielding is observed. These results are fundamentally different from the classical expectations for metallic glasses, in which the transition from discrete to continuous yielding occurs upon a decrease in deformation rate. The present experimental results are analysed with reference to the theoretical ideal-plastic strain field beneath an indenter and rationalized on the basis of mechanistic models of glass plasticity.
\end{abstract}

\section{$\S 1$. INTRODUCTION}

The plastic deformation of bulk amorphous metals is fundamentally different from that in crystalline solids, because of the lack of long-range order in the atomic structure. Whereas dislocations and defects carry plastic strain in crystals, amorphous alloys yield through intense shearing in narrow bands (Argon et al. 1985, Donovan 1988, Hays et al. 2000, Wright et al. 2001a, b, Kim et al. 2002). Mechanistically, these shear bands are self-organized assemblies of smaller units of plasticity, that is, volume elements of material containing about 30-50 atoms that individually undergo local shear transformation. Topologically, such shear motions require a local increase in free volume (a dilatation) and produce an elastic strain field that autocatalytically initiates similar shear transformations in neighbouring volume elements. Argon and co-workers have successfully modelled this phenomenon analytically (Argon 1979, Argon and Shi 1983), using two-dimensional (2D)

\footnotetext{
†Email: schuh@mit.edu.
} 
analogues (Argon and Kuo 1979, Argon and Shi 1982), as well as computer simulation techniques (Deng et al. 1989, Bulatov and Argon 1994).

The operation of shear bands on the atomic level gives rise to several unique mechanical properties at the macroscopic level. For example, in amorphous metals:

(i) macroscopic yield apparently cannot be described by the classic von Mises yield criterion (Davis and Kavesh 1975, Donovan 1989, Lowhaphandu et al. 1999, Flores and Dauskardt 2001, Vaidyanathan et al. 2001), which is broadly valid for most crystalline metals,

(ii) in tension, little or no macroscopic ductility is observed, because of separation along a single plane of shear (Megusar et al. 1979, Mukai et al. 2002a) and

(iii) in compression, plastic yielding is observed, but it does not occur smoothly, instead exhibiting 'load serrations' during brief intervals when the strain is carried by a single shear band (Kimura and Masumoto 1983, Wright et al. 2001a).

These novel properties have been investigated in a variety of amorphous metals with very different compositions, and appear to be general to this class of materials, which collectively are candidates for many structural and functional applications.

Instrumented nanoindentation is increasingly being used to probe the mechanical properties of a highly localized region in materials (Bhushan 1999). During indentation, the depth of a rigid indenter tip is monitored as it is pressed into a substrate; the resulting load-displacement response reflects deformation by plasticity and/or elasticity. In preliminary indentation studies on amorphous alloys, shear bands have been observed on the specimen surface and beneath the indenter (Golovin et al. 2001, Vaidyanathan et al. 2001, Kim et al. 2002). Additionally, the activation of shear bands under the indenter (as observed by atomic force microscopy) has been correlated with discrete displacement bursts that appear as discontinuities in the load-displacement curve (Golovin et al. 2001, Wright et al. 2001a, Greer and Walker 2002, Schuh et al. 2002, Schuh and Nieh 2003). When such bursts are observed in crystalline materials, they are ascribed to non-continuum events such as defect nucleation (Corcoran et al. 1997, Suresh et al. 1999, Gouldstone et al. 2001); as the depth of the indent increases, these discrete events give way to continuum plasticity in crystals, reflected in a smooth, parabolic load-displacement curve.

In the present work, nanoindentation is used to explore the nature of plasticity in a Pd-based bulk metallic glass. We identify an apparent incongruity with respect to the established mechanistic understanding of plastic flow in these alloys, where more rapid indentation leads to homogenization of flow. Combining existing models of glass plasticity and the flow field under an indenter, we offer a mechanistic explanation for the unique experimental observations.

\section{§2. EXPERIMENTAL PROCEDURES AND RESUlts}

The bulk metallic glass used in this work was an alloy of composition Pd-10 at.\% $\mathrm{Ni}-30$ at. $\% \mathrm{Cu}-20$ at. $\% \mathrm{P}$ prepared by a casting technique (Kato et al. 2001). The specimen was mechanically polished to a mirror finish and tested in a TriboIndenter instrumented nanoindenter (from Hysitron, Minneapolis, Minnesota), using constant loading rates from $8 \times 10^{-5}$ to $2 \times 10^{-2} \mathrm{~N} \mathrm{~s}^{-1}$, with peak applied loads of $10 \mathrm{mN}$. The constant-loading-rate experiment tends to exhibit a roughly constant 
indenter velocity, which ranged from $10^{-9}$ to $10^{-5} \mathrm{~m} \mathrm{~s}^{-1}$ in these tests. In all the experiments, a conical diamond indenter with a blunt spherical tip radius of $450 \mathrm{~nm}$ was used, and care was taken to ensure that the thermal drift of the instrument was maintained below $0.5 \mathrm{~nm} \mathrm{~s}^{-1}$. Displacement bursts occur quite rapidly; so data were acquired at sampling rates near $6000 \mathrm{~Hz}$ for most of the experiments reported here, and at somewhat lower rates (near $1000 \mathrm{~Hz}$ ) for some of the longer experiments.

Several typical load $P$-displacement $h$ curves are shown in figure 1 for the loading portion of the experiment; the origin of each curve has been displaced for clearer observation. For the indentations performed at lower loading rates, these curves are punctuated by many displacement bursts, where the indentation depth increases abruptly at an approximately constant load. The depth of individual discontinuities increases with increasing load or depth, as a consequence of the increasing length scale of the indentation geometry. As figure 1 illustrates, the character of the displacement bursts depends on the rate of loading; slow loading conditions produce sharper, more horizontal discontinuities. At higher loading rates, the discontinuities appear less as horizontal bursts than as gentle ripples in the load-displacement curve, each one extended over a range of loads. At the highest rate investigated $\left(0.33 \mathrm{~N} \mathrm{~s}^{-1}\right)$, the load-displacement curve is smooth and does not exhibit any notable discontinuities.

Owing to the very rapid data acquisition used in some of the present experiments, it was possible to measure the duration of individual shear localization events with reasonable accuracy. Figure 2 shows a histogram derived from analysis of 48 individual rapid displacement bursts, indicating the number of bursts with a given duration $\Delta \tau$. The modal burst duration was $6 \mathrm{~ms}$, with a spread of about $\pm 4 \mathrm{~ms}$. These values are in general agreement with the result of Neuhauser (1978), who identified for $\Delta \tau \approx 2-6 \mathrm{~ms}$ in cinematographic studies of shear band motion.

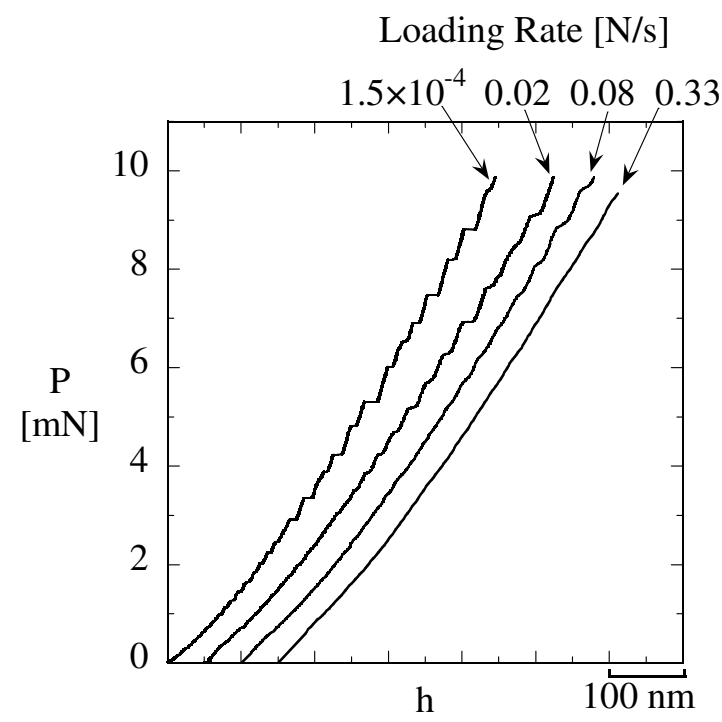

Figure 1. Applied load $P$ plotted against the nanoindentation depth $h$ for amorphous $\mathrm{Pd}-10$ at. $\% \mathrm{Ni}-30$ at. $\% \mathrm{Cu}-20$ at. $\% \mathrm{P}$, at four different loading rates. 


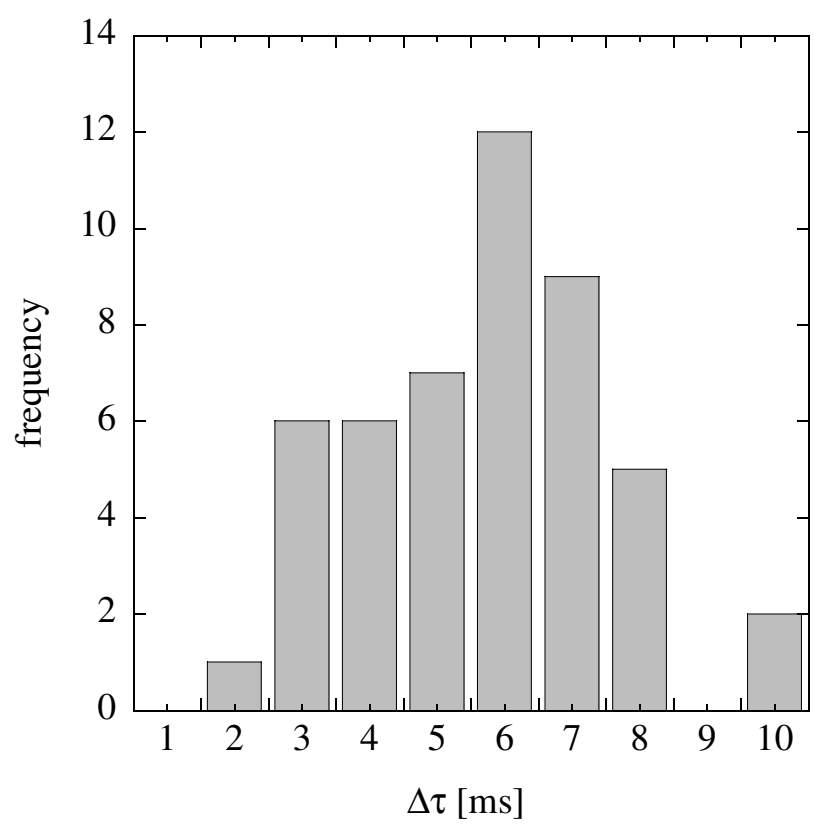

Figure 2. Histogram illustrating the distribution of rapid displacement burst durations $\Delta \tau$ during experiments such as shown in figure 1.

\section{§3. TRANSITION FROM DISCRETE TO HOMOGENEOUS PLASTICITY}

In figure 3, one example of a load-displacement curve at a slow loading rate $\left(8 \times 10^{-5} \mathrm{~N} \mathrm{~s}^{-1}\right)$ is shown; the depth recovered upon elastic unloading is denoted as $h_{\mathrm{e}}$. The contribution of discrete shear bands to the total elastic-plastic curve can be assessed by removing all the discontinuities from the curve, forming a 'consolidated elastic' load-displacement curve (Gouldstone et al. 2000). The arrows in figure 3 illustrate this procedure, and the resulting consolidated curve is a smooth bold curve. The maximum depth of the consolidated curve is $h_{\mathrm{e}}^{\prime} \approx 223 \mathrm{~nm}$, which is close to the true elastic depth of the indentation, $h_{\mathrm{e}} \approx 208 \mathrm{~nm}$. This result indicates that essentially all the plastic strain experienced by the amorphous alloy occurs in discretized displacement bursts, by the operation of individual shear bands.

The above result can be further validated by considering the expected theoretical consolidated elastic curve, for which the load-displacement relation is given by Oliver and Pharr (1992):

$$
\frac{\mathrm{d} P}{\mathrm{~d} h}=\frac{2}{\pi^{1 / 2}} E_{\mathrm{R}} A^{1 / 2}
$$

with $E_{\mathrm{R}}$ the reduced biaxial modulus $\uparrow$ and $A$ the projected contact area between indenter and substrate. When small strains and a spherical indenter are assumed,

$\dagger E_{\mathrm{R}}^{-1}=\left(1-v_{\mathrm{i}}\right) E_{\mathrm{i}}^{-1}+\left(1-v_{\mathrm{s}}\right) E_{\mathrm{s}}^{-1}$, where $E$ is the elastic modulus, $v$ is Poisson's ratio, and the subscripts $i$ and s denote the indenter and substrate respectively. In the present case we use $E_{\mathrm{R}}=104 \mathrm{GPa}$, based on constants from the work of Wang et al. (2000) and Golovin et al. (2001); the same value was obtained by analysis of elastic unloading curves in this work, based on the method given by Oliver and Pharr (1992). 


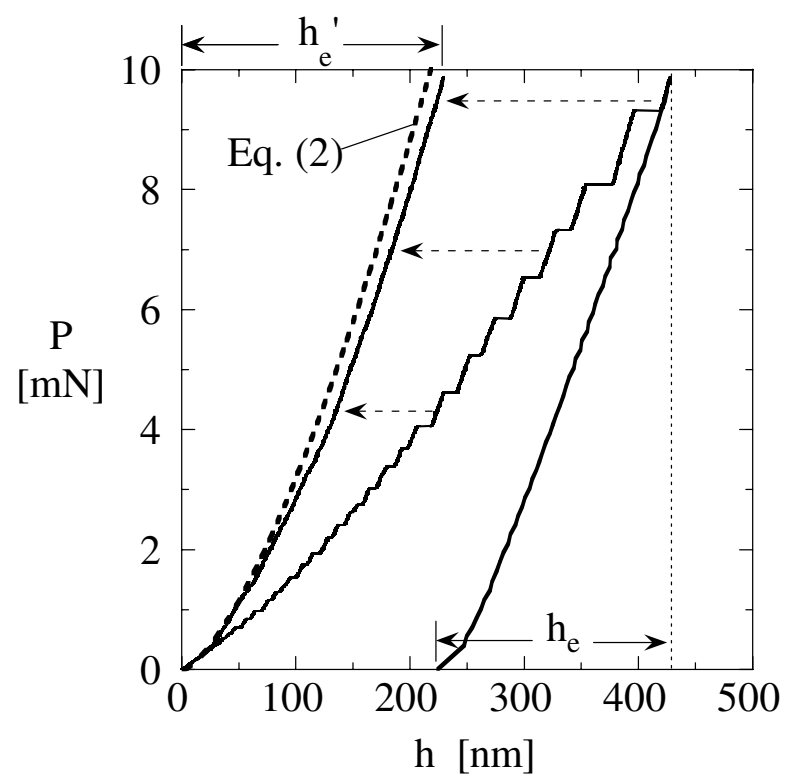

Figure 3. The serrated black curve illustrates the load $P$-displacement $h$ curve upon loading at a rate of $8 \times 10^{-5} \mathrm{~s}^{-1}$, followed by the elastic recovery to a depth $h_{\mathrm{e}}$ upon unloading. After removal of the plastic discontinuities, the corrected loading curve (smooth black curve denoted by the arrows) matches the contact mechanics prediction for pure elasticity (the broken curve predicted by equation (2)).

equation (1) reduces to the classic Hertz contact theory (Johnson 1985). For the present geometry, the indenter tip is approximately spherical for shallow indents, and equation (1) reduces to

$$
\frac{\mathrm{d} P}{\mathrm{~d} h_{\mathrm{e}}}=2 E_{\mathrm{R}}\left[2 R h_{\mathrm{p}} \Delta_{\mathrm{p}}-\left(h_{\mathrm{p}} \Delta_{\mathrm{p}}\right)^{2}\right]^{1 / 2},
$$

where $R$ is the radius of the indenter and the parameter $\Delta_{\mathrm{p}}$ is introduced as a correction factor that accounts for the elastic deflection of the surface around the indent $\dagger$. In figure 3, the elastic prediction found by integrating equation (2) is shown for $\mathrm{Pd}-10$ at. $\% \mathrm{Ni}-30$ at. $\% \mathrm{Cu}-20$ at. $\% \mathrm{P}$ as a broken curve, which is in close agreement with the consolidated elastic curve derived experimentally. This conformity between theory and experiment further confirms that the plastic deformation sustained during nanoindentation can be wholly attributed to displacement burst events. Although prior research has shown that shear band propagation produces plastic strain in amorphous alloys, the results in figure 3 indicate that, at sufficiently slow deformation rates, discretized shear band activity is the only source of plastic strain in this alloy.

$\dagger$ During nanoindentation, the projected contact area is a function of the depth of the indent. Because the surface of the specimen deflects elastically around the indent, the measured depth $h$ is somewhat larger than the true depth of the indent. Equation (2) for the consolidated elastic $P-h$ curve thus requires a correction, as discussed in detail by Oliver and Pharr (1992). The correction factor is taken as $\Delta_{\mathrm{p}}=1-\varepsilon / 2$, where $\varepsilon \approx 0.75$ as given by Oliver and Pharr (1992). 


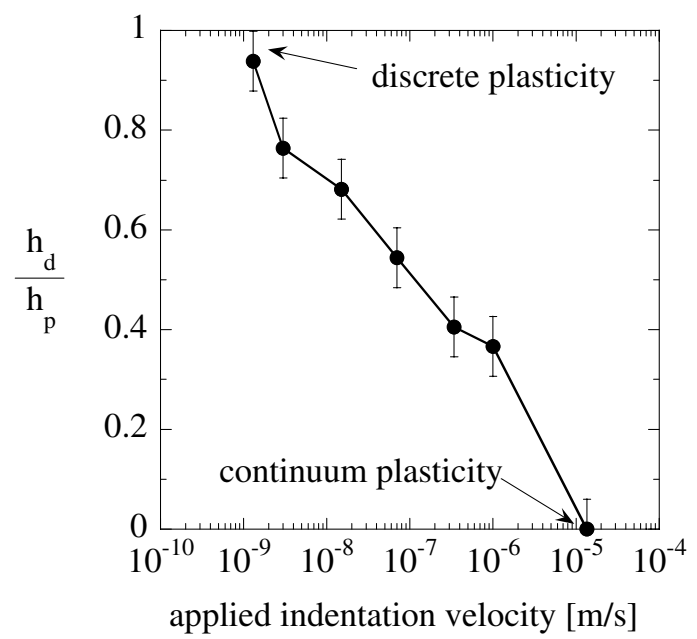

Figure 4. The ratio of discrete plastic depth $h_{\mathrm{d}}$ to plastic depth $h_{\mathrm{p}}$ as a function of nanoindentation velocity. This ratio approaches unity at low rates (corresponding to fully discretized plasticity), and zero at high rates (corresponding to smooth continuous yielding).

Just as the result of figure 3 suggests complete localization of plasticity at a low loading rate, the curves in figure 1 illustrate homogeneous plastic yielding at high loading rates, with no evidence of any discrete plasticity events. The transition from discretized- to continuum-yielding behaviour is summarized in figure 4, in which the fraction of the total plastic depth attributable to discrete displacement bursts is plotted as a function of the applied indentation velocity. The end points on this figure illustrate the two extremes described above, where all or none of the plastic deformation can be attributed to discrete yielding bursts. Over the four-decade range of indenter velocities between these endpoints, the data present a smooth transition from continuum to discrete plasticity.

\section{$\S 4$. Mechanistic interpretation}

The interesting aspect of the above experimental observations is their fundamental difference from the usual plastic response of metallic glasses. Whereas we report a transition from localized to homogeneous plasticity as the strain rate is increased, many prior studies have demonstrated that metallic glasses undergo shear localization at high strain rates but deform quite homogeneously at low strain rates or high temperatures above a characteristic temperature (Spaepen 1977, Argon 1979, Megusar et al. 1979). This classical behaviour is explained mechanistically by a flow-induced dilatation effect, where 'unit' plastic processes (local shear transformations in clusters of atoms incorporating some excess free volume) are accompanied by a topologically required activation dilatation (Spaepen 1977, Argon 1979). At elevated temperatures and/or low strain rates, diffusive rearrangements among atoms are rapid; so flow-induced dilatations collapse as rapidly as they form and deformation is homogeneous. At lower temperatures and/or high strain rates the local flow-induced dilatations (free volume) are not dispersed by local diffusive rearrangements, producing a drop of plastic shear resistance that autocatalytically results in intense shear localization (Argon 1979, Bulatov and Argon 1994). 
In tension experiments this produces runaway shearing off and separation by a characteristic Taylor meniscus instability fracture, which has been modelled as a ductile crack extension process (Argon and Salama 1976). In support of these models, flow dilatations inside intense shear bands of glassy metals have been measured ex post facto (Megusar et al. 1982, Argon et al. 1985), and recent experiments have shown that shear bands contain vacancy clusters (Li et al. 2002) or nanocrystals (Kim et al. 2002), both of which are consistent with an increase in free volume within shear bands.

At first glance, the experimental results described previously are at variance with the above mechanistic description of plasticity in metallic glasses. In what follows we consider the details of deformation beneath an indenter and show that the present results can, in fact, be interpreted on the same mechanistic basis.

\subsection{The plastic flow field under an indenter}

To analyse the reported behaviour at low indentation rates where intense shear localization occurs, some understanding of the plastic flow field is essential. We idealize the material response to be of two parts: a purely elastic Hertzian response and a plastic rigid non-hardening response as figure 3 suggests. An accurate flow field solution for such combined material behaviour is not available. A rigid-plastic slip line solution under a spherical indenter is available but is too complex to be of use in the present context (Ishlinsky 1944). To provide an approximate but still insightful analysis we treat the rigid-plastic response component by means of an axisymmetric slip-line field solution of a rigid frictionless cone impressed into a rigid-plastic non-hardening material half space (Lockett 1963). It is important to note that the slip-line field solution used below assumes that yield occurs on the surfaces of maximum shear although, for metallic glasses, yield apparently also involves a small pressure dependence (Lowhaphandu et al. 1999, Flores and Dauskardt 2001, Vaidyanathan et al. 2001). Given the assumed indenter shape and pressure-independent yield criterion, the developments to follow are intended only to be approximate and illustrative, and not a rigorous theory.

Figure 5 shows the classical flow field of Hill (1950) for a 2D plane-strain wedge indentation, made up of four right triangles and two focused shear fans connecting the triangles. For an axisymmetric cone indentation the field is qualitatively similar, although the slip lines and the pile-up surface are curved (Lockett 1963, Johnson 1985). We take this to be a reasonable representation of the plastic indentation field in its final stages. To create a semblance of similarity between the actual problem and the slip-line field solution, we assume that at any point during the indentation the true indenter can be approximated by an ideal cone of the same volume, for which the half-angle $\theta$ is given as

$$
\tan \theta=\left(\frac{3}{\pi h_{\mathrm{t}}^{3}} \int_{0}^{h_{\mathrm{t}}} A(h) \mathrm{d} h\right)^{1 / 2},
$$

where $A(h)$ is the empirical area function of the indenter tip (for example, Oliver and Pharr (1992)), and $h_{\mathrm{t}}$ is the total plastic depth of the indenter below its contact point with the surface, including the plastic depth $h_{\mathrm{p}}$ relative to the original undisturbed surface and the height $h_{\text {pile-up }}$, of the piled-up material above the original surface (see figure 5):

$$
h_{\mathrm{t}}=h_{\mathrm{p}}+h_{\text {pile-up }} \text {. }
$$




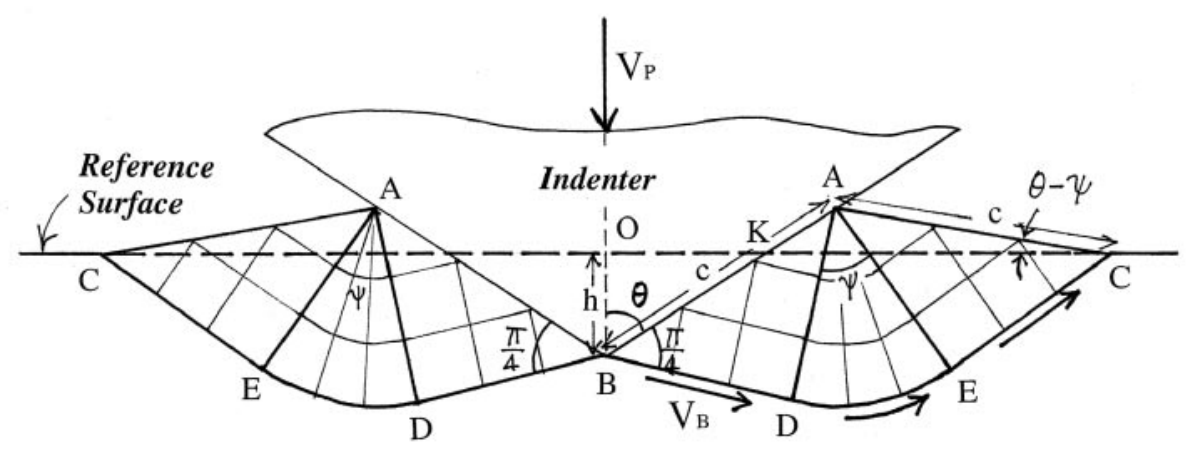

Amorphous metal

Figure 5. Idealized slip-line plastic field beneath a wedge-shaped indenter. Under axisymmetric flow the field is similar, but the material surfaces AC and slip lines exhibit some curvature.

In figure 5, the triangle $\mathrm{KAC}$ represents the piled-up material conserving that from the indented region BOK. At a given plastic depth $h_{\mathrm{p}}$, the current slip activity is concentrated entirely in the outer paths BDEC towards both sides, connecting the indenter tip to the free surface. The triangular zones and the focused shear fan have been sheared previously, and slip activity is dormant there. All parts of the half-space below the slip-line field in the amorphous metal are considered rigid, where the state of stress does not exceed yield. Additional geometric parameters, including the shear fan angle $\psi$ and the shape of the piled-up surface are derived as part of the full slip-line field solution.

Lockett (1963) has solved the cone indentation problem using a finite-difference slip-line field analysis, assuming that the indented material has a plastic shear resistance $k$. His results are summarized in figure 6, which plots the mean pressure applied to the indenter (i.e. the hardness) as a function of the cone half-angle $\theta$. These results are conveniently summarized by a linear least-squares fitted relationship:

$$
H=k(1.41+2.72 \theta) .
$$

The total plastic indentation load $P$ then becomes

$$
P=\pi h_{\mathrm{t}}^{2} \tan ^{2} \theta k(1.41+2.72 \theta) .
$$

As part of his numerical solution, Lockett (1963) also determined the height of the piled-up material around the indentation, as shown in figure 6. Again, his numerical results are reasonably expressed by a least-squares linear fit as

$$
\frac{h_{\text {pile-up }}}{h_{\mathrm{t}} \tan \theta}=(0.454-0.298 \theta) \text {. }
$$

All that is required to evaluate the above flow-field model is an estimate of the shear resistance $k$ of the $\mathrm{Pd}-30$ at. $\% \mathrm{Cu}-10$ at. $\% \mathrm{Ni}-20$ at. $\% \mathrm{P}$ metallic glass. Although we know of no direct measurement of this parameter for this alloy, there are some data that suggest a value of $k \approx 1 \mathrm{GPa}$. Firstly, uniaxial deformation experiments on similar glasses, including Pd-20 at.\% Ni, Pd-17 at. \% Si-6 at.\% Cu 


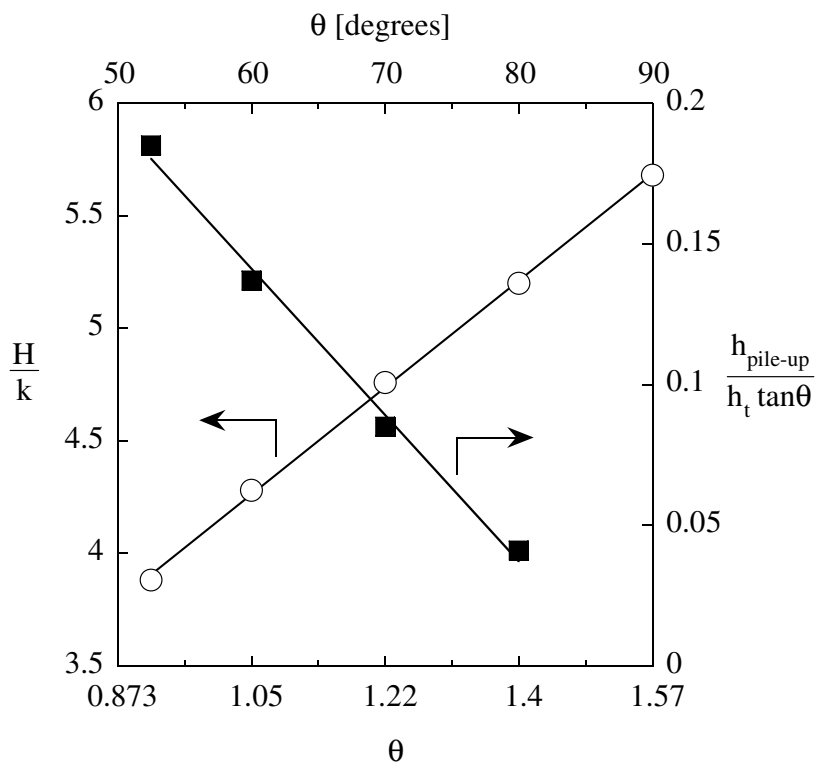

Figure 6. Summary of the slip-line field calculations of Lockett (1963), summarizing the changes in hardness and pile-up height (both non-dimensionalized) for an ideal conical indenter with included angle $\theta$. Least-squares fitted lines corresponding to equations (5) and (7) are also shown.

and $\mathrm{Pd}-40$ at. $\% \mathrm{Ni}-20$ at. $\% \mathrm{P}$, give the yield stress $\sigma_{\mathrm{y}} \approx 1.7 \mathrm{GPa}$ at nearly all loading rates (Chen 1973, Pampillo and Chen 1974, Donovan 1988, Mukai et al. 2002a, b). Using the von Mises relation between tensile and shear strength then gives $k \approx 0.98 \mathrm{GPa}$. Secondly, Golovin et al. (2001) have indented a glass with nominally the same composition as the present alloy, $\mathrm{Pd}-30$ at. $\% \mathrm{Cu}-10$ at. $\% \mathrm{Ni}-20$ at. $\% \mathrm{P}$, giving average hardness values of about $5 \mathrm{GPa}$. Using an approximate connection between hardness and tensile flow stress $\left(\mathrm{H} \approx 3 \sigma_{\mathrm{y}}\right)$ again gives $\sigma_{\mathrm{y}} \approx 1.7 \mathrm{GPa}$ and $k \approx$ $0.98 \mathrm{GPa}$. Based on the reported Young's modulus of $105 \mathrm{GPa}$ (Golovin et al. 2001) and an assumed Poisson's ratio of 0.3 for this alloy, we find that the ratio of shear resistance to shear modulus is $k / \mu=0.025$, which is quite in keeping with the expected ratio at room temperature of a typical amorphous metal alloy based on fundamental mechanistic models (Argon 1979).

In figure 7, the plastic indentation depth is plotted for the data set from figure 3, as are the predictions of the slip-line field model. The experimental data exhibit a staircase shape due to the perfectly discrete nature of plasticity in $\mathrm{Pd}-30$ at. $\% \mathrm{Cu}-$ 10 at. $\% \mathrm{Ni}-20$ at. $\% \mathrm{P}$ at this loading rate. Using as input $k=1 \mathrm{GPa}$, the model over-predicts the amount of plastic deformation in the glass, but the agreement with experiment is quite reasonable considering the many approximations made. It is particularly noteworthy that the model captures the linearity of the trend in figure 7, which results from the change in indenter geometry with depth. With a somewhat larger input of $k=1.5 \mathrm{GPa}$, a good quantitative agreement with experiment is obtained (figure 7). The larger value of $k$ required to fit the experimental data is probably associated with the various assumptions of the slip-line field solution that we have used; for example, a pressure-dependent shear resistance may account for 


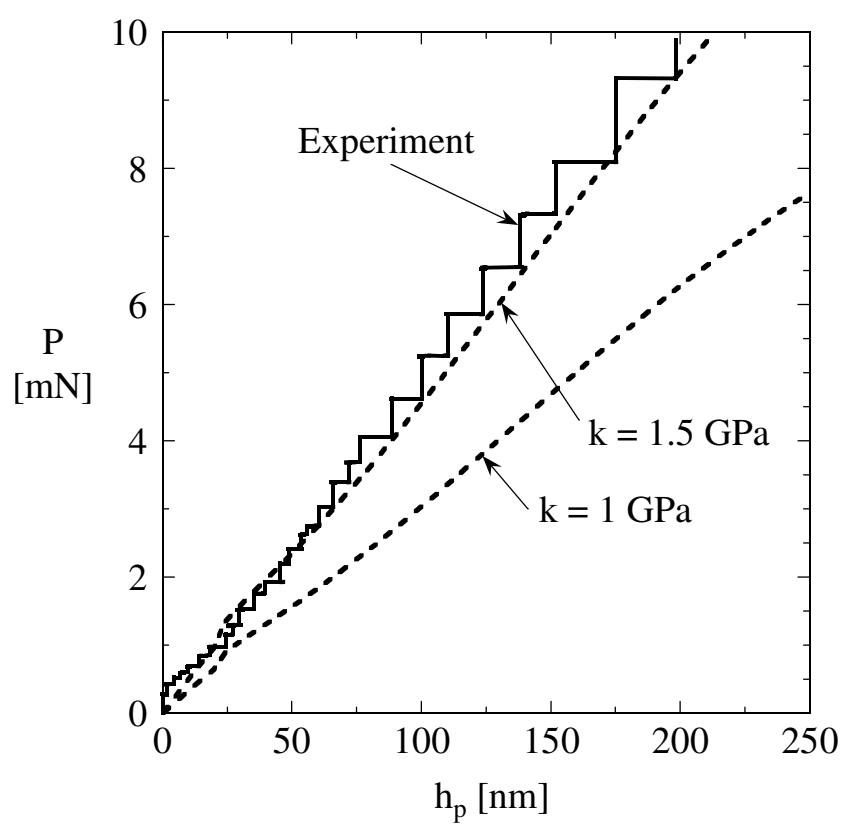

Figure 7. Comparison of the load $P$-plastic displacement $h_{\mathrm{p}}$ curves from experiment $(-)$ and the slip-line field model (-..-) for the plastic indentation of $\mathrm{Pd}-30$ at. $\% \mathrm{Cu}-$ 10 at. $\% \mathrm{Ni}-20$ at. $\%$ P. The experimental data are obtained from the curves in figure 3.

this discrepancy. In any case, we believe that at any given plastic depth the slip-line field model can be used as a reasonable illustration of the plastic flow field under the indenter.

With a description of the flow field in hand, the average slip velocity at the plastic zone border of BDEC, can be calculated as $V_{\mathrm{B}} \approx V_{\mathrm{i}} 2^{1 / 2} \sin \theta$, with $V_{\mathrm{i}}$ the velocity of the indenter. For the applied indentation velocities $V_{\mathrm{i}}=10^{-9}-10^{-5} \mathrm{~m} \mathrm{~s}^{-1}$, average slip velocities in the range from $1.1 \times 10^{-9}$ to $1.4 \times 10^{-5} \mathrm{~m} \mathrm{~s}^{-1}$ are suggested. However, during individual displacement bursts, the instantaneous indenter velocity is measured to be as high as $2 \times 10^{-5} \mathrm{~m} \mathrm{~s}^{-1}$. This value is also reflected by the data in figure 4 , which show that displacement bursts can no longer be observed above $V_{\mathrm{i}} \approx$ $10^{-5} \mathrm{~m} \mathrm{~s}^{-1}$. The local velocity of these discrete slip events (nominally the velocity of shear banding) is thus estimated as $V_{\mathrm{B}} \approx 1.1 \times 10^{-5}-2.8 \times 10^{-5} \mathrm{~m} \mathrm{~s}^{-1}\left(11-28 \mu \mathrm{m} \mathrm{s}^{-1}\right)$. These values of $V_{\mathrm{B}}$ are much too low to account for the fact that shear bands can propagate across macroscopic specimens on millisecond time scales (Wright et al. 2001b). This result implies that the autocatalytic nucleation of an embryonic shear band is slow compared with the subsequent propagation of the shear band, as expected on the basis of mechanistic models (Argon 1979). This viewpoint will be used in the following section to explain the observed transition from discrete to continuous yielding in the indentation experiments.

\subsection{Mechanism of the transition from localized to homogeneous flow}

For the Hertzian spherical contact problem it is known that plastic deformation is initiated at a distance of about half the contact radius beneath the indenter, when 
the deviatoric stress there reaches the tensile plastic resistance and the indentation load $P$ reaches a value

$$
P_{\text {crit }}=372 E a \frac{k^{3}}{E^{3}},
$$

where $a$ is the radius of the contact. On a more mechanistic basis, this initiation of plasticity occurs in a representative volume element (RVE) incorporating a small number of atomic clusters; the volume of the RVE we estimate from earlier studies to be about $7.7 \times 10^{-28} \mathrm{~m}^{3}$, that is roughly a diameter of $3-5 \mathrm{~nm}$ (Argon and Shi 1983 , Pekarskaya et al. 2001).

As discussed in considerable detail elsewhere (Argon 1979), all plastic flow in a glassy metal starts out at the length scale of the RVE and broadens homogeneously. At low temperatures where diffusive atomic rearrangements are too slow and flowinduced dilatations are sufficiently long lived to localize plastic flow autocatalytically, shear flow rapidly narrows into intense bands roughly of the thickness of the RVE (i.e. $3-5 \mathrm{~nm}$ ) at the expense of shear flow in the surroundings, which rapidly ceases. During this period, the specimen undergoes a macroscopic shear strain increment $\Delta \gamma \approx 0.01$ over a duration of the order of $4-10 \mathrm{~m} \mathrm{~s}$, as suggested by figure 2 and the cinematographic studies of Neuhauser (1978). Within the forming shear band there is a sharp dilatation-induced drop in plastic resistance by several per cent, while the surrounding material remains unsheared and stiff. Accordingly, the shear band soon acquires the character of a mode II crack with a nearly rate-independent shear resistance in its flanks (Argon and Salama 1976). It propagates very quickly, with a velocity approaching small fractions of the Rayleigh velocity.

Following the above discussion, we envisage the plastic response mode under the indenter as a time series of repeated plastic shear bursts, sweeping out along the borders BDEC (figure 5) toward the surface. For this discrete series of bursts to take place, the RVE of roughly $3 \mathrm{~nm}$ diameter must be subjected to homogeneous shear conditions for periods of $\Delta \tau \approx 6 \mathrm{~m} \mathrm{~s}$ (cf. figure 2 ), to nucleate an embryonic shear band. This translates into a critical indenter velocity of

$$
V_{\mathrm{i}, \mathrm{crit}}=\frac{3 \mathrm{~nm}}{6 \mathrm{~ms}}=5 \times 10^{-7} \mathrm{~m} \mathrm{~s}^{-1}
$$

for sampling of new RVEs under the indenter. If the applied indentation velocity is much slower than this value, then there is ample time for the autocatalytic activation of a shear band from the RVE. On the other hand, if the indentation velocity is much higher than this value, then the autocatalytic process to nucleate a shear band will be incomplete before the indenter has moved to sample different RVEs. As a consequence, we expect that high indenter velocities will maintain homogeneous flow. We imagine that, in this case, there is a field of shear transformations occurring in many RVEs to accommodate the applied strain, without a single discrete event of autocatalytic self-assembly into a shear band. A virtual field of less-correlated shear transformations then carries the plastic strain. Alternatively, one can envisage shear slip on many surfaces simultaneously as the indenter samples new RVEs with a rate higher than can be accommodated by a single shear band event. In the broadest sense, equation (9) gives a critical indentation rate beyond which the autocatalytic shear band formation step cannot be observed as a discrete event.

The critical indentation velocity calculated in equation (9) falls in the midrange of indentation velocities over which the discrete-continuous transition is observed 
$\left(10^{-9}-10^{-5} \mathrm{~m} \mathrm{~s}^{-1}\right.$ (figure 4)). The fact that this transition is observed experimentally over a broad range of strain rates is most probably due to the usual local structural variations in the amorphous metal alloy. Such variations allow significant changes in both the size of the RVE and the duration $\Delta \tau$ of the nucleation event; the velocity estimated in equation (9) can thereby vary over several orders of magnitude.

\section{§5. Summary AND CONClUSIONS}

Using instrumented nanoindentation on a glassy $\mathrm{Pd}-30$ at. $\% \mathrm{Cu}-10$ at. $\%$ $\mathrm{Ni}-20$ at. \% P alloy, we have identified a fundamental change in plastic deformation behaviour as the rate of indentation is increased across several orders of magnitude. At low deformation rates, plasticity occurs in discrete bursts, reminiscent of the effects observed in incipient crystal plasticity under a nanoindenter. In the case of amorphous alloys, these bursts are known to correlate with the operation of shear bands, which form beneath the indenter tip and progress to the surface in a few milliseconds. For our lowest indentation rate, we demonstrate that the entire plastic strain accumulated during nanoindentation is due to such discrete events. In contrast, higher rates do not show such prominent localization of plasticity, and only a fraction of the measured plastic indentation depth can be ascribed to discrete shear band events. At the highest indentation rates investigated, plasticity appears completely homogeneous in time, with no evidence for discrete events associated with shear banding.

The experimental observations have also been discussed with reference to an ideal rigid-plastic solution for the flow field under an indenter. Coupled with mechanistic theories for the initiation and propagation of plasticity in amorphous metals, this approach gives critical geometric and kinetic insight into the experimental results. The analysis implies that the nucleation of an embryonic shear band is the ratelimiting step in shear localization and suggests a critical indentation velocity above which this nucleation can no longer be observed as a discrete event. The predictions of the model are quantitatively reasonable with respect to the experimental data for $\mathrm{Pd}-30$ at. $\% \mathrm{Cu}-10$ at. $\% \mathrm{Ni}-20$ at. $\% \mathrm{P}$.

\section{ACKNOWLEDGEMENTS}

This work resulted within the context of a DURINT Program on nanostructured materials supported by the US Office of Naval Research under grant N00014-01-10808. A.S.A. acknowledges useful discussions with Professor D.M. Parks, and Dr Y. Kawamura is gratefully acknowledged for supplying the experimental material. Portions of this work were performed under the auspices of the US Department of Energy by the University of California, Lawrence Livermore National Laboratory under contract W-7405-Eng-48.

\section{REFERENCES}

Argon, A. S., 1979, Acta metall., 27, 47.

Argon, A. S., and Kuo, H. Y., 1979, Mater. Sci. Engng, 39, 101.

Argon, A. S., Megusar, J., and Grant, N. J., 1985, Scripta metall., 19, 591.

Argon, A. S., and Salama, M., 1976, Mater. Sci. Engng, 23, 219.

Argon, A. S., and Shi, L. T., 1982, Phil. Mag., A, 46, 275; 1983, Acta metall., 31, 499.

Bhushan, B., 1999, Handbook of Micro/Nano Tribology, edited by B. Bhushan (Boca Raton, Florida, CRC Press), p. 433.

Bulatov, V. V., and Argon, A. S., 1994, Modelling Simulation Mater. Sci. Engng, 2, 167.

Chen, H. S., 1973, Scripta metall., 7, 931. 
Corcoran, S. G., Colton, R. J., Lilleodden, E. T., and Gerberich, W. W., 1997, Phys. Rev. B, 55, R16057.

Davis, L. A., and Kavesh, S., 1975, J. Mater. Sci., 10, 453.

Deng, D., Argon, A. S., and Yip, S., 1989, Phil. Trans. R. Soc., 329, 613.

Donovan, P. E., 1988, Mater. Sci. Engng., 98, 487; 1989, Acta mater., 37, 445.

Flores, K. M., and Dauskardt, R. H., 2001, Acta mater., 49, 2527.

Golovin, Y. I., Ivolgin, V. I., Khonik, V. A., Kitagawa, K., and Tyurin, A. I., 2001, Scripta mater., 45, 947.

Gouldstone, A., Koh, H.-J., Zeng, K.-Y., Giannakopoulos, A. E., and Suresh, S., 2000, Acta mater., 48, 2277.

Gouldstone, A., VanVliet, K. J., and Suresh, S., 2001, Nature, 411, 656.

Greer, A. L., and Walker, I. T., 2002, Mater. Sci. Forum, 386-388, 77.

Hays, C. C., Kim, C. P., and Johnson, W. L., 2000, Phys. Rev. Lett., 84, 2901.

Hill, R., 1950, Plasticity (Oxford: Clarendon).

Ishlinsky, A., 1944, Prikl. Mate. Mek., 8, 201.

Johnson, K. L., 1985, Contact Mechanics (Cambridge, University Press).

Kato, H., Kawamura, Y., Inoue, A., and Chen, H.-S., 2001, Mater. Sci. Engng, A304-A306, 758.

Kim, J.-J., Choi, Y., Suresh, S., and Argon, A. S., 2002, Science, 295, 654.

Kimura, H., and Masumoto, T., 1983, Acta metall., 31, 231.

Li, J., Spaepen, F., and Hufnagel, T. C., 2002, Phil. Mag., A, 82, 2623.

Lockett, F. J., 1963, J. Mech. Phys. Solids, 11, 345.

Lowhaphandu, P., Montgomery, S. L., and Lewandowski, J. J., 1999, Scripta mater., 41, 19.

Megusar, J., Argon, A. S., and Grant, N. J., 1979, Mater. Sci. Engng, 38, 63; 1982, Rapidly Solidified Amorphous and Crystalline Alloys, edited by B. H. Kear, B. C. Giessen and M. Cohen (Amsterdam, Elsevier), p. 283.

Mukai, T., Nieh, T. G., Kawamura, Y., Inoue, A., and Higashi, K., 2002a, Scripta mater., 46, 43; 2002b, Intermetallics, 10, 1071.

Neuhauser, H., 1978, Scripta metall., 12, 471.

Oliver, W. C., and Pharr, G. M., 1992, J. Mater. Res., 7, 1564.

Pampillo, C. A., and Chen, H. S., 1974, Mater. Sci. Engng, 13, 181.

Pekarskaya, E., Kim, C. P., and Johnson, W. L., 2001, J. Mater. Res., 16, 2513.

Schuh, C. A., and Nieh, T. G., 2003, Acta mater., 51, 87.

Schuh, C. A., Nieh, T. G., and Kawamura, Y., 2002, J. Mater. Res., 17, 1651.

Spaepen, F., 1977, Acta metall., 25, 407.

Suresh, S., Nieh, T. G., and Choi, B. W., 1999, Scripta mater., 41, 951.

Vaidyanathan, R., Dao, M., Ravichandran, G., and Suresh, S., 2001, Acta mater., 49, 3781 .

Wang, L. M., Sun, L. L., Wang, W. H., Wang, R. J., Zhan, Z. J., Dai, D. Y., and Wang, W. K., 2000, Appl. Phys. Lett., 77, 3734.

Wright, W. J., SAHA, R., and Nix, W. D., 2001a, Mater. Trans. Japan Inst. Metals, 42, 642.

Wright, W. J., Schwarz, R. B., and Nix, W. D., 2001b, Mater. Sci. Engng, A319-A321, 229. 